\title{
Веденuе nauueнmов
}

\section{с единичнымu u редкимu эnuлепmuческимu npucmynaмu}

\author{
А.С. Котов \\ ГБУЗ МО «Московский областной научно-исследовательский клинический институт им. М.Ф. Владимирского»; \\ Россия, 129110 Москва, ул. Щепкина, 61/2 \\ Контакты: Алексей Сергеевич Котов alex-013@yandex.ru
}

\begin{abstract}
Цель исследования - изучение структуры заболевания и разработка тактики лечения пациентов с единичными и редкими эпилептическими приступами.

Материалы и методы. Было обследовано 1200 пациентов с эпилепсией, выявлено 103 пациента, перенесших не более 3 приступов в течение всей жизни. Обследование включало изучение анамнеза, клинический и неврологический осмотры, рутинную электроэнцефалографию и/или видеоэлектроэнцефалографический мониторинг, магнитно-резонансную томографию головного мозга.

Результаты. Рецидив приступов у пациентов с отслеженным катамнезом произошел в 32 \% случаев, с риском рецидива ассоциировались эпилептогенные изменения на томограмме и особенно анатомо-электроклиническая корреляция очага эпилепсии.

Выводы. Решение о медикаментозной терапии после первого неспровоцированного приступа (или единичных приступов) должно базироваться на соотношении риска повторных приступов и риска побочных эффектов терапии. Это решение должно быть индивидуальным и учитывать не только медицинские проблемы, но и предпочтения пациента и членов его семьи.
\end{abstract}

Ключевые слова: эпилепсия, эпилептический приступ, редкие эпилептические приступы, риск рецидива приступов

Для цитирования: Котов А.С. Ведение пациентов с единичными и редкими эпилептическими приступами. Русский журнал детской неврологии 2020;15(2):12-6.

DOI: $10.17650 / 2073-8803-2020-15-2-12-16$

(cc) BY

\section{MANAGEMENT OF PATIENTS WITH SINGLE AND RARE EPILEPTIC SEIZURES}

\author{
A.S. Kotov \\ M.F. Vladimirsky Moscow Regional Research Clinical Institute; 61/2 Shchepkina St., Moscow 129110, Russia
}

Objective: to study the structure of the disease and develop tactics of treatment of patients with single and rare epileptic seizures.

Materials and methods. 1200 patients with epilepsy were examined, 103 patients were identified who had no more than 3 seizures throughout their life. Study included evaluation of anamnesis, clinical and neurological examination, routine electroencephalography and/or videoelectroencephalographic monitoring, magnetic resonance imaging of the brain.

Results. Relapse of seizures in individuals with a history of follow-up occurred in $32 \%$ of cases; epileptogenic changes in magnetic resonance image and, especially, anatomical and electro-clinical correlation of the epilepsy focus were associated with the risk of relapse.

Conclusions. The decision on drug therapy after the first unprovoked seizure should be based on the ratio of the risk of repeated seizures and the risk of side effects. This solution should be individual and take into account not only medical problems, but also the preferences of the patient and his family members.

Key words: epilepsy, epileptic seizure, rare seizures, risk of seizures relapse

For citation: Kotov A.S. Management of patients with single and rare epileptic seizures. Russkiy zhurnal detskoy nevrologii = Russian Journal of Child Neurology 2020;15(2):12-6. (In Russ.).

\section{Введение}

Ранее считалось, что почти все пациенты, попавшие в поле зрения врачей с единственным эпилептическим приступом, неизбежно будут иметь последующие приступы, и многие из них получали лечение антиэпилептическими препаратами (АЭП) [7, 14]. Кроме того, основываясь на данных исследований, проведенных в локальных консультативных центрах, эпилепсию относили к хроническим, прогрессирующим и неизлечимым заболеваниям $[8,16]$. Таким образом, раннее начало агрессивной терапии АЭП должно было быть выгодным с точки зрения подавления заболевания или контроля над его прогрессированием. Результаты недавних эпидемиологических и рандомизированных клинических исследований значительно изменили понимание природы приступов и эпилепсии. 
Целью нашего исследования было изучение структуры заболевания и разработка тактики лечения пациентов с единичными и редкими эпилептическими приступами.

\section{Матершалы u методы}

Из 1200 пациентов, обратившихся за специализированной помощью к эпилептологу, были отобраны 410, которые, по данным представленной медицинской документации, перенесли за всю историю болезни к моменту наблюдения не более 3 эпилептических приступов. Среди отобранных пациентов было 193 мужчины и 217 женщин в возрасте от 19 до 84 лет. Обследование включало клинический и неврологический осмотры, рутинную электроэнцефалографию (ЭЭГ) и/или видео-ЭЭГ-мониторинг (ВЭМ), магнитно-резонансную томографию (МРТ) головного мозга, лабораторные анализы. Всем нуждающимся в этом пациентам была назначена впервые или подвергнута коррекции текущая терапия АЭП. Катамнез сроком от 6 мес до 10 лет (в среднем 3 года) отслежен у 303 больных.

Статистическую обработку данных проводили в программе Excel 2016 (Microsoft, США). Для качественных переменных рассчитывали абсолютные и относительные частоты. Частоту признаков сравнивали путем ранжирования.

\section{Результаты и обсужденше}

После обследования у 126 (30,7 \%) пациентов из 410 были выявлены частые приступы (более 3 приступов в год). Причиной этому служило отсутствие осознания врачами и больными простых (и, в ряде случаев, сложных) фокальных приступов, а также абсансов и/или миоклоний как эпилептических феноменов. Так, в нашем исследовании было выявлено 120 пациентов с идиопатической генерализованной эпилепсией, у 56 (46,7 \%) из них констатированы частые эпилептические приступы.

Один эпилептический приступ в анамнезе имели 17 (4,1 \%) больных, 2 приступа перенесли 45 (11\%), 3 приступа - 41 (10 \%), остальные 307 (74,9 \%) пациентов до начала исследования перенесли более 3 приступов, как удалось установить после детального сбора анамнеза.

Для оценки влияния различных факторов на риск повторения приступов были отобраны 103 пациента, перенесшие на момент включения в исследование не более 3 приступов. Терапия АЭП была рекомендована всем пациентам, кроме перенесших один приступ и не имеющих эпилептиформной активности по данным ЭЭГ/ВЭМ и эпилептогенных структурных аномалий по данным МРТ $(n=12)$.

У остальных пациентов $(n=91)$ эпилептогенные и условно эпилептогенные структурные изменения по данным МРТ были выявлены лишь в 14 случаях, эпилептиформная активность по данным ЭЭГ/ВЭМ в 17. Анатомо-электроклиническая корреляция (совпадение семиотики приступов, локализации региональной эпилептиформной активности по данным ЭЭГ с эпилептогенным структурным дефектом по данным МРТ) отмечалась лишь у 5 больных.

На момент завершения исследования катамнез прослежен у 5 из 12 пациентов, которым не были назначены АЭП; рецидив приступов произошел лишь у 1 из них. Можно предположить, что остальные пациенты (утраченные для катамнестического наблюдения) также не имели приступов, поэтому отказались от посещений врача.

Из группы тех, кому были рекомендованы АЭП $(n=91)$, катамнез удалось отследить у 69; эпилептические приступы в отобранной группе отмечены в $32 \%$ случаев.

В данной когорте пациентов с высоким риском рецидива приступов ассоциировалась эпилептогенность структурных повреждений мозга, выявленных при проведении МРТ (вероятность приступов была в 2,7 раза выше, чем у лиц без патологических находок или с неэпилептогенными изменениями по данным МРТ). Наличие и характер эпилептиформной активности, обнаруженной при проведении ЭЭГ, не продемонстрировали влияния на риск рецидива приступов, что, очевидно, связано с недостаточной информативностью рутинной ЭЭГ. Для большей информативности среди лиц, перенесших 1-3 приступа, было отобрано 34 пациента, которым проводился ночной ВЭМ. Однако и в этой когорте не выявлено взаимосвязи между наличием и характером эпилептиформной активности по данным ЭЭГ и риском рецидива приступов, что, очевидно, объясняется небольшим числом наблюдений и отсутствием патологии по данным ВЭМ в большинстве случаев.

Из 5 пациентов с анатомо-электроклинической корреляцией очага эпилепсии рецидив приступов произошел во всех случаях.

В конце исследования была изучена переносимость терапии: об отсутствии или незначительности побочных эффектов заявили 73,6 \% пациентов из числа получающих АЭП, выраженные побочные эффекты отмечались у 18,9 \%, отмена АЭП из-за побочных эффектов произошла у 7,5\% пациентов.

Взгляд на прогноз при эпилепсии претерпел существенные изменения за последние полвека. До конца 1960-х годов считалось, что ремиссии смогут достичь не более $1 / 3$ пациентов с эпилепсией, а у большинства (до $85 \%$ ) заболевание будет хроническим [16]. Кроме того, считалось, что приступы порождают приступы. Как писал один из «отцов эпилептологии» W.R. Gowers: «Когда один приступ произошел, будь он следствием какого-либо немедленного возбуждения или нет, последующие приступы обычно следуют за ним без 
какой-либо непосредственной причины. Воздействие приступа на нервные центры приводит к тому, что последующие приступы происходят более легко, приступ активизирует причину, которая уже существует. Таким образом, можно сказать, что любой приступ является следствием предшествующих приступов и причиной последующих» [8]. Однако популяционные и проспективные исследования, проведенные за последние 30 лет, свидетельствуют, что до 90 \% пациентов с впервые диагностированной эпилепсией достигают длительной ремиссии и около 40-60 \% из них избавляются от приступов сразу после начала лечения [10].

Хотя лучшие результаты недавних исследований можно было бы объяснить улучшением лечения, эпидемиологические данные показывают, что плохой прогноз в ранних исследованиях объяснялся «смещением отбора» - в поле зрения попадали в основном резистентные пациенты, направленные из региональных медицинских учреждений [3, 14], в то время как недавние исследования включали пациентов с впервые диагностированной эпилепсией.

Прежде подразумевалось, что лечение эпилепсии должно начинаться как можно раньше, потому что заболевание является текущим процессом и чем позже начато лечение, тем выше риск развития резистентности к АЭП [14]. Это предположение подтверждалось экспериментальной киндлинг-моделью эпилепсии у животных [15].

Альтернативная точка зрения заключается в том, что исход эпилепсии в основном зависит от причины, лежащей в основе заболевания, а лечение на течение эпилепсии не влияет $[17,18]$. Полученные нами данные согласуются с данной концепцией.

У пациентов, обратившихся к врачу по поводу первого неспровоцированного приступа, риск рецидива составляет от 23 до $71 \%$ [2, 4]. Ширина этого диапазона отражает значительные различия между исследованиями по длительности периода наблюдения, целевой выборке, определению понятия «первый приступ» и дизайну исследования [3, 4].

Популяционные исследования дают более однородные результаты: риск рецидива в течение года составляет 36-37\%, в течение 2 лет - 43-45 \% [1,9]. Полученные нами данные соответствуют этим результатам - рецидив приступов был констатирован в $32 \%$ случаев.

Большое мультицентровое исследование FIRST включало 397 детей и взрослых в возрасте от 2 до 70 лет. Целью исследования было установление влияния лечения первого приступа на риск рецидива и долгосрочный прогноз эпилепсии. Пациентов с первым тонико-клоническим приступом с фокальным началом или без последнего, произошедшим в течение предшествующих 7 дней и подтвержденным свидетелями, распределяли в группы, получающие карбамазепин, фенитоин, фенобарбитал, вальпроаты, или в группу, не получающую терапии. В целом, 36 из 204 леченых и 75 из 193 нелеченых пациентов имели рецидив приступов. Относительный риск рецидива для нелеченых больных составил 2,8 (95\% доверительный интервал 1,9-4,2). Эффект от терапии сохранялся несмотря на то, что 41 (20\%) леченый пациент в то или иное время прекратил прием АЭП [13].

Результаты этого исследования были подтверждены в ходе другого крупного рандомизированного исследования MESS, целью которого было сравнение немедленного и отсроченного начала лечения дебюта эпилепсии и единичных приступов. В данном открытом мультицентровом исследовании пациенты в возрасте старше 1 мес подвергались рандомизации, если у пациента и врача не было уверенности в необходимости немедленного начала лечения. В результате 722 пациента были рандомизированы в группу немедленного лечения и 721 - в группу отсроченного лечения, из них 404 и 408 пациентов соответственно имели на момент рандомизации 1 приступ. Немедленное начало лечения удлиняло время до повторного приступа (относительный риск 1,5; 95 \% доверительный интервал $1,2-1,8)$ и увеличивало число пациентов с 2 -летней ремиссией (64 и $52 \%$ соответственно, $p=0,023$ ) [12].

Эти данные согласуются с другими сообщениями и подтверждают предположение о том, что лечение первого неспровоцированного эпилептического приступа существенно влияет на краткосрочный прогноз, снижая риск повторных приступов независимо от возраста пациента, этиологии приступов и находок при проведении ЭЭГ [5, 6]. Однако исследования FIRST и MESS также показали, что долгосрочный прогноз эпилепсии (достижение 2-летней ремиссии после рандомизации) практически не зависит от лечения первого приступа.

Таким образом, лечение первого неспровоцированного приступа, очевидно, способно снизить риск рецидива во время активной фазы заболевания, но не влияет на долгосрочный прогноз эпилепсии. Другими словами, АЭП оказывают симптоматическое воздействие на приступы, но не лечебный эффект на течение эпилепсии, определяемое конкретным эпилептическим синдромом, что согласуется с полученными нами данными.

В 2017 г. Международной противоэпилептической лигой была предложена новая версия классификации эпилепсии, согласно одному из положений которой для дефиниции «эпилепсия» следует оценить риск повторения приступов в течение ближайших 10 лет. К сожалению, абсолютно достоверных предикторов течения заболевания не существует. В нашем исследовании с риском рецидива ассоциировались эпилептогенные изменения по данным МРТ. Результаты ЭЭГ были менее однозначными, так как информативность рутинной ЭЭГ у взрослых невысока, а ночной ВЭМ 
могли выполнить не все пациенты (да и он у лиц, перенесших единичные приступы, часто не выявляет эпилептиформную активность). В связи с вышеизложенным решение о назначении терапии АЭП после единственного приступа (или редких приступов) должно базироваться на данных клиники (например, если приступы повторяются 1 раз в 5 лет, то, скорее всего, в дальнейшем они будут следовать с примерно такой же частотой), ЭЭГ и МРТ (которые следует проводить всем лицам, перенесшим первый в их жизни эпилептический приступ), а также обязательно учитывать желание пациента (поскольку для одних пациентов приступы неприемлемы, и они готовы годами принимать АЭП, а для других приступ 1 раз в год кажется меньшим злом, чем ежедневный прием препаратов).

\section{Выводы}

В свете накопленных эпидемиологических данных, изменивших наше представление о приступах и эпилепсии, лечение после первого приступа уже не назначается автоматически. Необходимо учесть множество факторов, в том числе влияние лечения на рецидив приступов, долгосрочный прогноз эпилепсии, побочные эффекты (в том числе тератогенность) и стоимость лекарств.

Стратегия ведения взрослых пациентов после первого неспровоцированного приступа подробно описана в современных рекомендациях Американской академии неврологии [18]. Согласно этим рекомендациям врачу следует сообщить пациенту о том, что риск рецидива приступов максимален в течение первых 2 лет после первого приступа и составляет 21-45 \% (уровень доказательности А). К факторам, повышающим риск рецидива, относятся предшествующее повреждение мозга (уровень доказательности А), эпилептиформная активность по данным ЭЭГ (уровень доказательности А), клинически значимые изменения при нейровизуализации (уровень доказательности В) и возникновение первого приступа во время сна (уровень доказательности В). Немедленное назначение лечения после первого приступа по сравнению с задержкой начала терапии до второго приступа может снизить риск рецидива приступов в течение ближайших 2 лет (уровень доказательности В), но не повысить качество жизни (уровень доказательности С). Немедленное назначение терапии АЭП на срок более 3 лет не улучшит долгосрочный прогноз в отношении избавления от приступов (уровень доказательности В). Пациентам следует сообщить, что риск побочных эффектов терапии составляет 7-31 \%, при этом в большинстве случаев эти побочные эффекты будут легкими/обратимыми (уровень доказательности В).

Решение о медикаментозной терапии после первого неспровоцированного приступа должно базироваться на соотношении риска от повторных приступов и риска побочных эффектов терапии. Это решение должно быть индивидуальным и учитывать не только медицинские проблемы, но и предпочтения пациента и членов его семьи.

\section{ЛИТ Е PAT У PA/REFERENGES}

1. Annegers J.F., Shirts S.B., Hauser W.A., Kurland L.T. Risk of recurrence after an initial unprovoked seizure. Epilepsia 1986;27(1):43-50. DOI: $10.1111 /$ j.1528-1157.1986. tb03499.x.

2. Beghi E., Ciccone A. Recurrence after a first unprovoked seizure. Is it still a controversial issue? First Seizure Trial Group (first). Seizure 1993;2(1):5-10. DOI: 10.1016/s1059-1311(05)80096-2.

3. Berg A.T., Shinnar S. The contributions of epidemiology to the understanding of childhood seizures and epilepsy. J Child Neurol 1994;9(Suppl 2):19-26.

4. Berg A.T., Shinnar S. The risk of seizure recurrence following a first unprovoked seizure: a quantitative review. Neurology 1991;41(7):965-72. DOI: 10.1212/wnl.41.7.965.

5. Chandra B. First seizure in adults: to treat or not to treat. Clin Neurol Neurosurg
1992;94(Suppl):S61-3 DOI: 10.1016/0303-8467(92)90024-w.

6. Das C.P., Sawhney I.M., Lal V., Prabhakar S. Risk of recurrence of seizures following single unprovoked idiopathic seizure. Neurol India 2000;48(4):357-60.

7. Elwes R.D., Johnson A.L., Reynolds E.H. The course of untreated epilepsy. BMJ 1988 15;297(6654):948-50. DOI: $10.1136 / \mathrm{bmj} .297 .6654 .948$.

8. Gowers W.R. Epilepsy and other chronic convulsive diseases. London: Churchill, 1881.

9. Hart Y.M., Sander J.W., Johnson A.L., Shorvon S.D. National General Practice Study of Epilepsy: recurrence after a first seizure. Lancet 1990;336(8726):1271-4. DOI: 10.1016/0140-6736(90)92960-p.

10. Jallon P. Prognosis of epilepsies. Montrouge: John Libbey Eurotext, 2003.

11. Krumholz A., Shinnar S., French J. et al. Evidence-based guideline: Management of an unprovoked first seizure in adults: Report of the Guideline Development Subcommittee of the American Academy of Neurology and the American Epilepsy Society. Neurology 2015;85(17):1526-7. DOI: 10.1212/01.wnl.0000473351.32413.7c.

12. Marson A., Jacoby A., Johnson A. et al. Immediate versus deferred antiepileptic drug treatment for early epilepsy and single seizures: a randomized controlled trial. Lancet 2005;365(9476):2007-13. DOI: 10.1016/S0140-6736(05)66694-9.

13. Randomized clinical trial on the efficacy of antiepileptic drugs in reducing the risk of relapse after a first unprovoked tonicclonic seizure. First Seizure Trial Group (FIR.S.T. Group). Neurology 1993;43(3 Pt 1):478-83. DOI: $10.1212 /$ wnl.43.3_part_1.478.

14. Reynolds E.H. Early treatment and prognosis of epilepsy. Epilepsia 
1987;28(2):97-106

DOI: $10.1111 /$ j.1528-1157.1987.tb03633.x.

15. Reynolds E.H. The process of epilepsy:

is kindling relevant? In: The Clinical

Relevance of Kindling. Eds.: G. Bowling,

M.R. Trimble. Chichester: John Wiley

\& Sons, 1989. Pp. 149-160.
16. Rodin E.A. The prognosis of patients with epilepsy. Springfield:

Charles C. Thomas, 1968.

17. Sander J.W. Some aspects of prognosis in the epilepsies: a review. Epilepsia 1993;34(6):1007-16.

DOI: $10.1111 / \mathrm{j} .1528-1157.1993 . t b 02126 . x$.
18. Shinnar S., Berg A.T.

Does antiepileptic drug therapy prevent the development of "chronic" epilepsy? Epilepsia 1996;37(8):701-8. DOI: $10.1111 / \mathrm{j} .1528-1157$. 1996.tb00639.x.

ORCID автора / ORCID of author

A.C. Котов / A.S. Kotov: https://orcid.org/0000-0003-2988-5706

Конфликт интересов. Автор заявляет об отсутствии конфликта интересов.

Conflict of interest. The author declares no conflict of interest.

Финансирование. Исследование проведено без спонсорской поддержки.

Financing. The study was performed without external funding.

\section{Соблюдение прав пациентов и правил биоэтики}

Протокол исследования одобрен комитетом по биомедицинской этике ГБУЗ МО «Московский областной научно-исследовательский клинический институт им. М.Ф. Владимирского».

Все пациенты подписали информированное согласие на участие в исследовании.

Compliance with patient rights and principles of bioethics

The study protocol was approved by the biomedical ethics committee of M.F. Vladimirsky Moscow Regional Research Clinical Institute.

All patients gave written informed consent to participate in the study. 